\title{
cDNA cloning and localization of Sp3111 (also called Ms4a14) in the rat testis
}

\author{
Yan $\mathrm{Xu}^{1, *}$, Miao Liu ${ }^{1, *}$, Yi-hua Gu${ }^{4}$, Xiao-feng Jia ${ }^{4}$, Yong-Mei Chen ${ }^{5}$, Michelle Santos ${ }^{2}$, \\ Ai-Zhen $\mathrm{Wu}^{2}$, Xiao-dong Zhang ${ }^{4}$, Hui-Juan Shi ${ }^{1,4}$ and Ching-Ling C Chen ${ }^{2,3,+}$ \\ ${ }^{1}$ Medical School of Fudan University, Shanghai, China, ${ }^{2}$ Center for Biomedical Research, Population Council, \\ New York, New York, USA, ${ }^{3}$ The Rockefeller University, New York, New York, USA, ${ }^{4}$ National Population and Family \\ Planning Key Laboratory of Contraceptive Drugs and Devices, Shanghai Institute of Planned Parenthood Research, \\ Shanghai, China and ${ }^{5}$ Department of Anatomy, Histology and Embryology, Institute of Basic Medical Sciences, \\ Chinese Academy of Medical Sciences, Beijing, China
}

Correspondence should be addressed to C-L C Chen; Email: chencl@aol.com or to H-J Shi; Email: shihuijuan2011@163.com

(Y Xu and M Liu contributed equally to this work)

${ }^{+} \mathrm{C}-\mathrm{L}$ C Chen is now at Spectronix Systems, Inc., Scarsdale, New York, USA

\begin{abstract}
With tetraspanning topology, members of the membrane-spanning four-domain subfamily A (MS4A) may facilitate signaling or ion channel functions in many tissues. In this study, we report the cloning of a full-length cDNA from rat testis, designated Ms4a14 (Sp3111), which encodes the MS4A protein with 1139 amino acid residues. In situ hybridization and immunohistochemical analyses indicate that Ms4a14 is predominantly expressed from round spermatids to spermatozoa at specific stages in the rat testis at both the mRNA and protein level. Immunofluorescence analysis revealed that MS4A14 (SP3111) is located in the acrosome and the midpiece of the flagellum in mature sperm. Previously, we explored and reported the involvement of MS4A14 in reproductive functions, using antibody blockage during IVF and a transgenic RNA interference method in a mouse model. Our results suggested that MS4A14 is involved in fertilization and zygote division. As MS4A14 protein exists in mammals, such as humans, cows, dogs, and rodents, MS4A14 may play a ubiquitous role in mammalian reproduction.
\end{abstract}

Reproduction (2014) $\mathbf{1 4 8} 81-86$

\section{Introduction}

Sperm-oocyte interaction involves a set of successive processes that include binding of sperm-zona pellucida, the penetration of sperm through the zona pellucida, and sperm-oocyte plasma membrane adhesion and fusion. Mammalian spermatozoa have a highly regionalized plasma membrane, as demonstrated by the numerous different binding patterns of the surface molecules. The cellular events of fertilization depend on the molecules present on the surface of the sperm head, probably through proteins both on the sperm surface and within the acrosome (Naz 2004, Stein et al. 2004, Naz et al. 2005). Proteomic analysis has revealed that over 100 proteins are expressed on mature sperm at the site of sperm-oocyte interactions. The functions of some of these proteins have been evaluated with respect to male reproduction using a gene knockout strategy, which indicated that at least nine proteins are related to male reproduction and two proteins are involved in embryonic development (Stein et al. 2006). Although the events implicated in this procedure are not fully understood at the molecular level (Yanagimachi et al. 1994), the sperm surface molecules are apparently necessary for successful sperm-oocyte interaction.

With one or more tetraspanning domains, membranespanning four-domain subfamily A (MS4A) consists of more than 16 members (Ishibashi et al. 2001). Some MS4A members are reported to have roles in tissues by functioning as ion channels or forming complexes with other cell surface proteins to participate in signal transduction. MS4A1 (also named CD20) in B lymphocytes and MS4A12 in colonic epithelial cells regulate calcium influx (Bubien et al. 1993, Koslowski et al. 2008), whereas MS4A2 (also referred as FCRB) on mast cells is a subunit of the high-affinity IgE receptor (FCER1) or the low-affinity IgG receptor (FCGR3) (Dombrowicz et al. 1998). MS4A5 (also called TETM4) and MS4A13 (also called NYD-SP21) are testis-specific proteins and are believed to associate with other cell surface proteins and participate in signaling events (Hulett et al. 2001). We cloned, from rat testis, a full-length 
cDNA, designated Ms4a14, with a high degree of sequence identity with Ms4a13. Sequence analysis suggested that the putative protein sequence of MS4A14 (SP3111) has 1139 amino acid residues, which contain a four-transmembrane domain and a glycoprotein domain. Our study indicates that MS4A14 is also a testis-specific protein. Although three members of the MS4A family, MS4A5, MS4A13, and MS4A14, have been found to be specifically expressed in the testis, studies on their functions have not yet been reported, especially with regard to male reproduction.

In this study, we report the isolation, tissue distribution, and localization of a new gene, designated Ms4a14, that encodes a novel member of the MS4A family and that is mainly expressed in rat testis.

\section{Materials and methods \\ Animals}

Sprague-Dawley rats aged 7-90 days were purchased from Charles River Breeding Laboratories (Wilmington, MA, USA). The use of animals was approved by the Rockefeller University Animal Care and Use Committee.

\section{$5^{\prime}$ - and $3^{\prime}$-RACE methods for the generation of a full-length Ms4a14 cDNA}

The $5^{\prime}$ end of the Ms4a14 cDNA was amplified using a GeneRacer Kit (Invitrogen) as described previously (Shi et al. 2004). The first-strand cDNA was synthesized by RT using 80-day-old rat testicular total RNA as a template. The Ms4a14 cDNA was then amplified by PCR using a 44-mer oligonucleotide provided in the kit and a 27-mer oligonucleotide, GSP1 (5'-GGCTGCCAGGATGGAGTTGACCAGAAG-3'), which was complementary to the nucleotide sequence from 2730 to 2756 of the predicted protein XM_219591 derived from rat chromosome 11. The $3^{\prime}$ end of the Ms4a14 cDNA was amplified using a 25-mer GeneRacer $3^{\prime}$ primer provided in the kit and a 26-mer oligonucleotide, GSP2 (5'-CTCCACACCGAGAGCCATCGTACCAA-3'), which was complementary to the nucleotide sequence from 1199 to 1224 in the predicted protein XM_219591. The PCR products were subcloned into the pCR4-TOPO plasmid using a TOPO TA Cloning Kit (Invitrogen), and their nucleotide sequences were analyzed.

\section{Northern blot and RT-PCR analyses}

Ten micrograms of each poly(A) RNA isolated from different-aged rat testes and other tissues was subjected to northern blot analysis. Newly isolated Ms4a14 cDNA was used to prepare radioactive probes for the identification of Ms4a14 mRNA on RNA blots.

Primers (upstream 5'-ACTAAGTAACTGCGAAGGGCTCAT$3^{\prime}$ and downstream 5'-ACAGTTTACATGAGATTTA-3') were used to prepare full-length Ms4a14 cDNA. RT was carried out using $2 \mu \mathrm{g}$ each of rat testicular total RNA sample. The cDNAs were amplified for 30-35 cycles of PCR with the following amplification cycle: $94{ }^{\circ} \mathrm{C}$ for $30 \mathrm{~s}, 57^{\circ} \mathrm{C}$ for $30 \mathrm{~s}$, and $68{ }^{\circ} \mathrm{C}$ for $4 \mathrm{~min}$. The RT-PCR products were analyzed by gel electrophoresis and were subcloned into the pCR4-TOPO vector for nucleotide sequence analysis.

\section{In situ hybridization analysis}

Following fixation, deproteination, and acetylation, testicular tissue slides were hybridized with antisense and sense RNA probes for the detection of Ms4a14 mRNA in the testis. RNA probes were prepared with digoxigenin-UTP (DIG-UTP) by in vitro transcription with SP6 and T7 RNA polymerase using a DIG RNA Labeling Kit (Roche Molecular Biochemicals). Sections were incubated with RNA probes overnight at $55^{\circ} \mathrm{C}$ and then washed with high stringency buffer that contained $50 \%$ formamide and $2 \times$ SSC. The DIG-labeled RNA probes that bound to testicular sections were detected by a color reaction upon incubation with anti-DIG antibody overnight at $4{ }^{\circ} \mathrm{C}$, as described (Shi et al. 2004). At least ten testicular sections were evaluated.

\section{Western blot analysis}

A synthetic peptide of 14 amino acids (SSYQRDQQQYEDSD) at the C-terminus of MS4A14 (1126-1139) (Anaspec Corporation, San Jose, CA, USA) was conjugated with KLH and used to immunize rabbits to produce antibody sera. Antibody concentrations were determined by ELISA test. The Econo-Pac Serum IgG Purification Kit (Bio-Rad Laboratories) was used to purify the $\lg G$ fraction of the polyclonal Ab2438 antibody.

Proteins were isolated from multiple tissues of 63-day-old rats using CytoBuster Protein Extraction Reagent (Novagen, Gibbstown, NJ, USA). Cauda epididymal sperm were freshly prepared by a swim-up method to obtain mainly motile, uncapacitated, and acrosome-intact cells (Hanada \& Chang 1972). Tissue and sperm samples were lysed using RIPA buffer, and protein concentrations were determined using the RC DC Protein Assay Kit (Bio-Rad). Twenty micrograms of each protein sample was fractionated on a $10 \%$ SDS-polyacrylamide gel and transferred onto a PVDF membrane (Millipore, Billerica, MA, USA). Antibody Ab2438 was used for detection at a dilution of 1:400, followed by incubation with a secondary antibody (HRPlinked anti-rabbit IgG). The MS4A14 protein was visualized using an $\mathrm{ECL}+$ plus detection system (Amersham Biosciences).

\section{Immunohistochemical and immunofluorescence staining}

Sections were deparaffinized in xylene and hydrated gradually through graded ethanol. Slides were treated at $95^{\circ} \mathrm{C}$ for $10 \mathrm{~min}$ in antigen unmasking solution (Vector $\mathrm{H}-3300$ ) and incubated for $10 \mathrm{~min}$ in $3 \%$ hydrogen peroxide to quench endogenous peroxidase activity. After blocking with $10 \%$ normal goat serum, the sections were incubated with antibody Ab2438, followed by incubation with a biotinylated secondary antibody. Visualization of positive signals was performed using a VectaStain Elite Kit (Vector Laboratories, Inc., Burlingame, CA, USA) according to the manufacturer's protocols.

For immunofluorescence staining, Alexa Fluor 488 goat antirabbit $\lg \mathrm{G}(\mathrm{H}+\mathrm{L})$ (Molecular Probes, Eugene, OR, USA) was 
added as a secondary antibody for $1 \mathrm{~h}$ at room temperature. The slides were counterstained with $300 \mathrm{nM}$ DAPI for $5 \mathrm{~min}$ and mounted using SlowFade light antifade mountain solution (Molecular Probes). Signals were viewed immediately using a fluorescence microscope.

Preimmune serum and antibody A2438 that had been preincubated with an excess of a synthetic oligopeptide of MS4A14 (amino acids 1126-1139) for $2 \mathrm{~h}$ at room temperature were used as negative controls. In general, 10-20 sections were evaluated for each stage.

\section{Results}

\section{Isolation and characterization of Ms4a14 cDNA from adult rat testis}

PCR products with the expected sizes of 2.4 and $2.9 \mathrm{~kb}$ were generated from 80-day-old rat testes by the $3^{\prime}$ and $5^{\prime}$-RACE method respectively. These overlapping DNA fragments were isolated and analyzed by nucleotide sequencing and used to prepare primers for the isolation of a 3.8-kb full-length Ms4a 14 cDNA from the rat testis. The nucleotide sequence of the full-length cDNA, designated Ms4a14, was submitted to GenBank under accession number NM_181082. The Ms4a14 cDNA consists of $120 \mathrm{bp}$ of the $5^{\prime}$-UTR, $3420 \mathrm{bp}$ of the coding region, with an open-reading frame of 1139 amino acids, and $261 \mathrm{bp}$ of the $3^{\prime}$-UTR. The amino acid sequence of the Ms4a14 cDNA-derived protein can be accessed through the NCBI Protein Database under accession number NP_851596.

\section{Sequence analysis of MS4A14 protein}

Within the amino acid sequence deduced from Ms4a14 cDNA, ExPASy proteomics tools (http://expasy.org/tools/) identified a leucine zipper pattern (amino acids 38-59), an ATP/GTP-binding site (amino acids 147-154), a glutamine-rich region (amino acids 401-1036), a threonine-rich region (amino acids 478-673), and a bipartite nuclear targeting sequence (amino acids 782-812), as well as several potential phosphorylation and glycosylation sites. The TMHMM program (http:// www.cbs.dtu.dk/services/TMHMM/), which predicts transmembrane helices in proteins, predicted four strong transmembrane helices in MS4A14, which are likely to adopt a membrane topology with both extracellular $\mathrm{N}$ - and C-termini (Fig. 1A). Furthermore, the NCBI Conserved Domain Database suggests that the MS4A14 protein contains a four-transmembrane domain and belongs to the gene superfamily named MS4A. The MS4A superfamily shares no homologies with other tetraspanin superfamily members, although all have four-transmembrane domains. The Ms4a14 gene has thus been named Ms4a14, which is also expressed in humans, the dog, cow, mouse, and other mammalian species. By using the Conserved Domain Architecture Retrieval Tool (CDART) (Geer et al. 2002), we found that
A

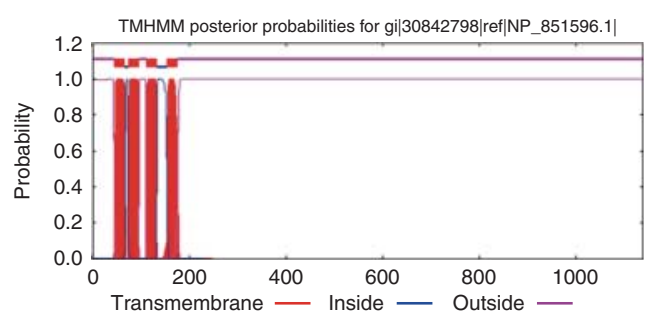

B

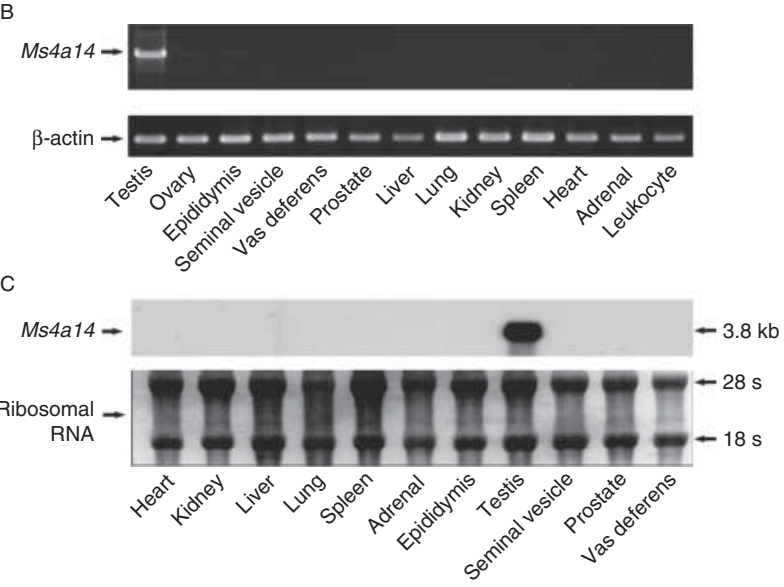

Figure 1 The predicted transmembrane structure of the MS4A14 protein and the tissue-specific expression of the Ms4a14 mRNA in adult rats. (A) The predicted transmembrane helices in MS4A14, (B) RT-PCR analysis for tissue-specific mRNA expression profile of Ms4a14, and (C) northern blot analysis for tissue-specific mRNA expression profile of Ms4a14. Total RNA ( $2 \mu \mathrm{g} /$ lane) isolated from various tissues of 63 -dayold adult rats was analyzed, $\beta$-actin mRNA was used to monitor RNA loading (B). Poly(A) RNA (10 $\mu$ g each) isolated from various tissues of 63-day-old male rats $(C)$.

MS4A14 is twice as long as other MS4A superfamily members, with an extended extracellular sequence at the C-terminus. As it contains some predicted phosphorylation and glycosylation sites, this protein may have signal transduction or cellular interaction functions.

\section{Expression pattern of the Ms4a14 gene}

To investigate the expression profile of the Ms4a14 gene in rats, RT-PCR analysis was carried out in 11 tissues of 63-day-old rats, with primers containing nucleotide sequences at positions of 819-848 and 3284-3313 of the Ms4a14 cDNA (Fig. 1B). Among the tissues examined, a single band of 2.5-kb DNA was detected at a high level in the testis, and a faint band of the same size was found in the epididymis or ovary: no other tissues expressed the Ms4a14 gene. The results were confirmed by northern blot analysis, and a single species of $3.8-\mathrm{kb}$ Ms4a14 mRNA was identified exclusively in the rat testis and was absent in other tissues of adult male rats (Fig. 1C).

We also generated an anti-MS4A14 polyclonal antibody, Ab2438. Protein extracts prepared from various tissues of 63-day-old rats were subjected to western blot 


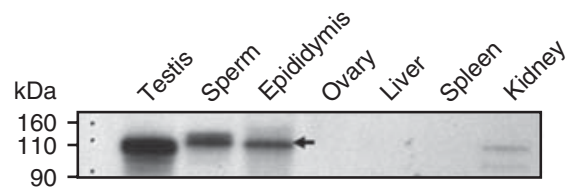

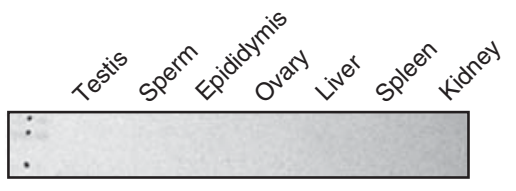

Figure 2 Western blot analysis of MS4A14 protein in various tissues of 63-day-old rats using the newly generated antibody Ab2438 (A) or preimmune serum (B). Protein extracts (20 $\mu \mathrm{g} / \mathrm{lane})$ were subjected to western blot analysis. An expected 127-kDa protein that immunoreacted with antibody Ab2438 is indicated by an arrow.

analysis. Ab2438 could detect a protein band with the molecular weight of $127 \mathrm{kDa}$ (Fig. 2), which is the theoretical molecular weight for the full-length MS4A14 protein, in the testis, epididymis, and cauda sperm. Because Ms4a14 mRNA was not identified in the epididymis (Fig. 1), MS4A14 protein in spermatozoa from the epididymis probably originates from the testis.

Because the Ms4a14 mRNA is mainly expressed in the testis, we studied the expression pattern of the Ms4a14 mRNA in developing rat testes, ranging from 7 to 90 days. The Ms4a14 cDNA generated by RT-PCR was first evident at 28 days and remained at high levels throughout adulthood (Fig. 3A). This finding was also confirmed by northern blot analysis (Fig. 3B). The agedependent expression of Ms4a14 mRNA was also demonstrated in mouse testes. A 4.1-kb mRNA that hybridized with Ms4a14 cDNA was detected in 28- and 75 -day-old mouse testes, but was not found at 21 days mouse. Our observation of the absence of testicular Ms4a14 mRNA expression at age $\leq 21$ days suggested that this gene begins to be expressed during the process of spermatogenesis after birth.

\section{Cellular and subcellular localization of Ms4a14 mRNA and MS4A14 protein in rat testis}

Testicular cell types that express Ms4a14 mRNA were identified by in situ hybridization using DIG-labeled antisense and sense RNA probes (Fig. 4A). The Ms4a14 mRNA was predominantly localized to round and elongated spermatids. The highest level of expression was observed at stages VII-VIII of round spermatids and stages IX-XI of early elongated spermatids (Fig. 4A, a, b and c). No apparent staining was detected when a sense RNA probe was used as a negative control (Fig. 4A, d).

We then localized the MS4A14 protein in the rat testis and epididymal sperm by immunohistochemical analysis using antibody Ab2438 for detection (Fig. 4B and C). Similarly to Ms4a14 mRNA, strong immunostaining of the MS4A14 protein was predominantly localized to round spermatids at stages VII-VIII and elongated spermatids at stages IX-XI, both in the testis (Fig. $4 \mathrm{~B}, \mathrm{a}, \mathrm{b}$ and $\mathrm{C}$ ). No staining was detected when preimmune serum was used (Fig. 4B, d).

Given that Ab2438 was generated against a synthetic 14-mer oligopeptide which was highly conserved between rat and mouse, we next examined the distribution of MS4A14 protein in both rat and mouse sperm isolated from the cauda epididymis (Fig. 4C). Using antibody Ab2438 for detection, intense fluorescent staining was observed at both the acrosomal region of the sperm head and the midpiece of the sperm tail (Fig. 4C, a and b). No staining was observed with preimmune serum in the sperm collected from the same animal (Fig. 4C, c).

\section{Discussion}

In the present study, we report the cDNA isolation of a novel gene in adult rat testes, Ms4a14, which is $3.8 \mathrm{~kb}$ long and encodes a protein with a molecular weight of $127 \mathrm{kDa}$. Conserved domain analysis revealed that the MS4A14 protein has a four-transmembrane domain and belongs to the MS4A subfamily. Among the 11 rat tissues examined, Ms4a14 mRNA expression is dominantly observed in rat testes in an age-dependent manner. Both in situ hybridization and immunological approaches indicate that MS4A14 is expressed in the round and elongated spermatids of adult rat testes. The MS4A14 protein in mature sperm was detected at the acrosomal region of the sperm head and at the midpiece of the sperm tail with intense immunofluorescent staining.

On the basis of the predicted conserved domains in the MS4A14 protein and the specific expression characteristics of Ms4a14 gene, our laboratory investigated the functional roles of MS4A14 in sperm motility and the sperm-egg reaction using an antibody raised against MS4A14 (Ab2438) (Jia et al. 2010). Our results demonstrated that, during the IVF procedure, pretreatment of

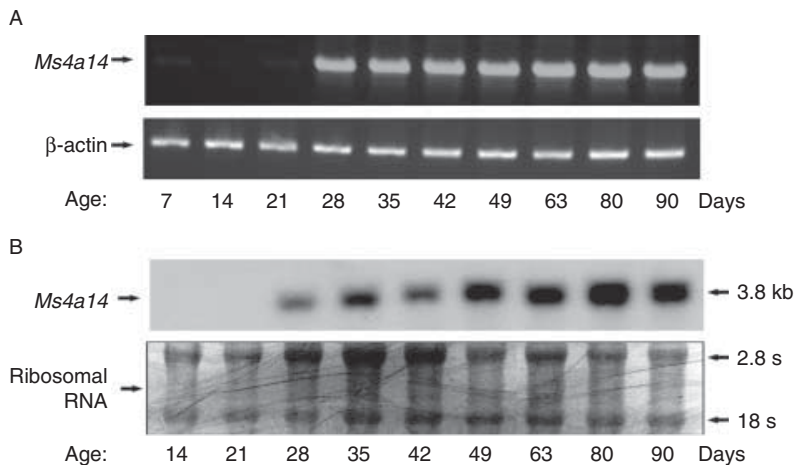

Figure 3 Age-dependent expression of the Ms4a14 mRNA in rat testes examined by RT-PCR (A) and by northern blot analysis (B), as described above in Fig. 1. 
A

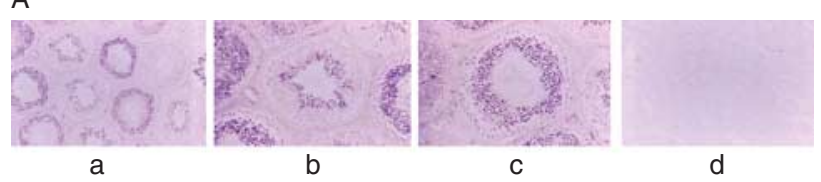

B

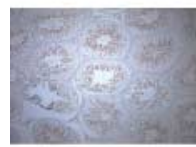

a

C

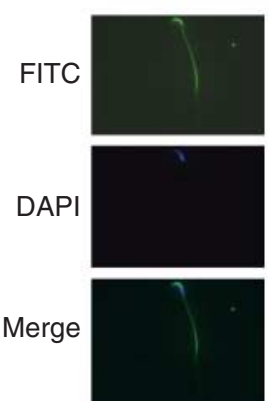

a

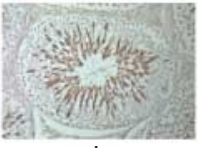

b

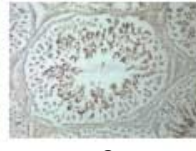

C
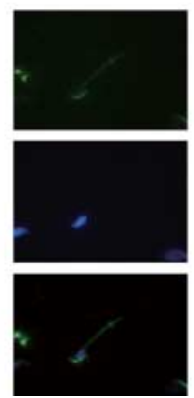

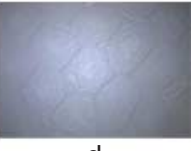

d
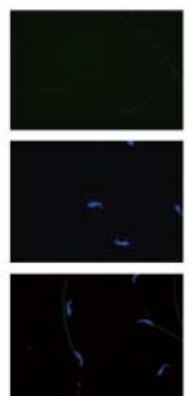

C
Figure 4 Localization of the Ms4a14 mRNA and MS4A14 protein in the rat testis and sperm. (A) Localization of the Ms4a14 mRNA in the 60-day-old rat testis by in situ hybridization with a digoxigenin-labeled cRNA (a, b and c) or sense RNA probe (d). (B) Immunohistochemical localization of MS4A14 protein in the adult rat testis using antibody Ab2438 (1:200 dilution) ( $a, b$ and c) or serum preabsorbed with a synthetic peptide (d). Magnification for both (A) and (B): $100 \times$ in (a) and (d), and 400 $X$ in (b) and (c). (C) Immunofluorescent localization (FITC) of MS4A14 protein in rat (a) and mouse (b) cauda epididymal sperm by using the Ab2438 antibody or serum preabsorbed with synthetic peptide (c). An intense FITC signal was detected on the anterior portion of the head and midpiece of all cells in (a) and (b). The DAPI staining of the rat and mouse sperm, and the merged picture of FITC and DAPI staining is shown below. Magnification: $400 \times$.

mature sperm with the antibody caused an evident reduction in the normal fertilization rate as well as a profound increase in zygote fragmentation. In addition, we have generated a transgenic RNA interference mouse model, in which the level of testicular Ms4a14 mRNA was lowered by $30 \%$. In vivo fertilization using transgenic males with WT females resulted in a reduced number of litters, and the fertility rate declined to $34.48 \%$ compared with $70.95 \%$ in the control group (Shi et al. 2010).

MS4A members including MS4A14 share only a same topology of transmembrane domain, but with a low sequence similarity and a diversity of functions. At least 26 other members of the MS4A family have been described in humans, mice, and rats. The members of MS4A family are expressed in various tissues, but are mostly expressed in lymphoid tissues such as the spleen, thymus, and intestines. The TETM4 gene is the first identified testisspecific member of the MS4A family; however, the localization of this protein on mature sperm and its function during fertilization has not been clearly elucidated thus far (Hulett et al. 2001). Similar to TETM4, the functions of most members of the MS4A family remain unknown. Unlike other members, MS4A14 has a much longer extracellular C-terminal tail, which possesses phosphorylation and glycosylation sites. Generally, membrane proteins with a large extracellular portion engage in cell contact or signal transduction. CD9 and CD81, two tetraspanins containing a large extracellular loop, have been reported to participate in signal transduction in the testis and during sperm-egg fusion function as a scaffold to bring other proteins together (Chen et al. 1999, Rubinstein et al. 2006). Our studies indicate that MS4A14 is also expressed in the testis and sperm of rats and mice, and blocking sperm with anti-MS4A14 antibody decreases the sperms' fertilization capability, suggesting that MS4A14 may have a similar role as CD9 and CD81 in the testis and during fertilization.

Although MS4A14 has been demonstrated to have functional importance in sperm function and fertilization in our studies by using molecular biology, immunohistochemistry, antibody blocking, and protein knockdown in vivo approaches, more studies are required to solidify our findings. For instance, immune electron microscopy or confocal microscopy is needed to confirm that MS4A14 is a sperm surface protein, and a gene knockout model could more clearly elucidate the function of MS4A14 in vivo.

\section{Declaration of interest}

The authors declare that there is no conflict of interest that could be perceived as prejudicing the impartiality of the research reported.

\section{Funding}

This work was supported in part by The Andrew W. Mellon Foundation, the National Natural Science Foundation of China (No. 30872765), and the Basic Research Key Program of Shanghai (10JC1409900).

\section{Acknowledgements}

The authors are grateful to late Dr Matthew Phillip Hardy for his critical discussions and consistent encouragement at the beginning of this study.

\section{References}

Bubien JK, Zhou LJ, Bell PD, Frizzell RA \& Tedder TF 1993 Transfection of the CD20 cell surface molecule into ectopic cell types generates a $\mathrm{Ca}^{2+}$ conductance found constitutively in B lymphocytes. Journal of Cell Biology 121 1121-1132. (doi:10.1083/jcb.121.5.1121)

Chen MS, Tung KS, Coonrod SA, Takahashi Y, Bigler D, Chang A, Yamashita Y, Kincade PW, Herr JC \& White JM 1999 Role of the integrinassociated protein CD9 in binding between sperm ADAM 2 and the egg integrin a6ß1: implications for murine fertilization. PNAS 96 11830-11835. (doi:10.1073/pnas.96.21.11830) 
Dombrowicz D, Lin S, Flamand V, Brini AT, Koller BH \& Kinet JP 1998 Allergy-associated FCR $\beta$ is a molecular amplifier of IgE- and IgGmediated in vivo responses. Immunity 8 517-529. (doi:10.1016/S10747613(00)80556-7)

Geer LY, Domrachev M, Lipman DJ \& Bryant SH 2002 CDART:, protein homology by domain architecture. Genome Research 12 1619-1623. (doi:10.1101/gr.278202)

Hanada A \& Chang M 1972 Penetration of zona-free eggs by spermatozoa of different species. Biology of Reproduction 6 300-309.

Hulett MD, Pagler E, Hornby JR, Hogarth PM, Eyre HJ, Baker E, Crawford J, Sutherland GR, Ohms SJ \& Parish CR 2001 Isolation, tissue distribution, and chromosomal localization of a novel testis-specific human fourtransmembrane gene related to CD20 and FcepsilonRI- $\beta$. Biochemical and Biophysical Research Communications 280 374-379. (doi:10.1006/ bbrc.2000.4088)

Ishibashi K, Suzuki M, Sasaki S \& Imai M 2001 Identification of a new multigene four-transmembrane family (MS4A) related to CD20, HTm4 and $\beta$ subunit of the high-affinity IgE receptor. Gene 264 87-93. (doi:10. 1016/S0378-1119(00)00598-9)

Jia XF, Zhou M, Lin JF, Shi WL, Zhang XD \& Shi HJ 2010 Role of SP3111 protein in fertilization and early embryo development in mice. Zhonghua Nan Ke Xue 16 14-19.

Koslowski M, Sahin U, Dhaene K, Huber C \& Tureci O 2008 MS4A12 is a colon-selective store-operated calcium channel promoting malignant cell processes. Cancer Research 68 3458-3466. (doi:10.1158/00085472.CAN-07-5768)

Naz RK 2004 Modalities for treatment of antisperm antibody mediated infertility: novel perspectives. American Journal of Reproductive Immunology 51 390-397. (doi:10.1111/j.1600-0897.2004.00174.x)

Naz RK, Gupta SK, Gupta JC, Vyas HK \& Talwar AG 2005 Recent advances in contraceptive vaccine development: a mini-review. Human Reproduction 20 3271-3283. (doi:10.1093/humrep/dei256)
Reddy KV, Vijayalaxmi G, Rajeev KS \& Aranha C 2006 Inhibition of spermegg binding and fertilisation in mice by a monoclonal antibody reactive to $57-k D a$ human sperm surface antigen. Reproduction, Fertility and Development 18 875-884. (doi:10.1071/RD06028)

Rubinstein E, Ziyyat A, Prenant M, Wrobel E, Wolf JP, Levy S, Le Naour F \& Boucheix C 2006 Reduced fertility of female mice lacking CD81. Developmental Biology 290 351-358. (doi:10.1016/j.ydbio.2005. 11.031)

Shi HJ, Wu AZ, Santos M, Feng ZM, Huang L, Chen YM, Zhu K \& Chen CL 2004 Cloning and characterization of rat spermatid protein SSP411: a thioredoxin-like protein. Journal of Andrology 25 479-493. (doi:10. 1002/j.1939-4640.2004.tb02819.x)

Shi W, Shi T, Chen Z, Lin J, Jia X, Wang J \& Shi H 2010 Generation of sp3111 transgenic RNAi mice via permanent integration of small hairpin RNAs in repopulating spermatogonial cells in vivo. Acta Biochimica et Biophysica Sinica 42 116-121. (doi:10.1093/abbs/gmp110)

Stein KK, Primakoff P \& Myles D 2004 Sperm-egg fusion: events at the plasma membrane. Journal of Cell Science 117 6269-6274. (doi:10.1242/jcs.01598)

Stein KK, Go JC, Lane WS, Primakoff P \& Myles DG 2006 Proteomic analysis of sperm regions that mediate sperm-egg interactions. Proteomics 6 3533-3543. (doi:10.1002/pmic.200500845)

Yanagimachi R, Knobil E \& Neill JD 1994 Mammalian Fertilization: University of Sheffield Library.

Received 15 February 2014

First decision 5 March 2014

Revised manuscript received 29 March 2014

Accepted 2 April 2014 